\title{
Comparison of thoracic epidural analgesia and thoracic paravertebral block in pain management after thoracotomy
}

\author{
$₫$ Musa Zengin, @Ramazan Baldemir, @Gülay Ülger, $₫$ Hilal Sazak, $₫$ Ali Alagöz \\ University of Health Sciences, Ankara Atatürk Chest Diseases and Thoracic Surgery Training and Research Hospital, Anesthesiology and Reanimation Clinic, \\ Ankara, Turkey
}

Cite this article as: Zengin M, Baldemir R, Ülger G, Sazak H, Alagöz A. Comparison of thoracic epidural analgesia and thoracic paravertebral block in pain management after thoracotomy. Anatolian Curr Med J 2022; 4(1); 70-75.

\begin{abstract}
Aim: Thoracotomy is among the most painful surgical incisions and causes severe acute pain in the postoperative period. Many analgesic methods have been suggested for thoracotomy pain treatment. Thoracic epidural analgesia (TEA) and thoracic paravertebral block (TPVB) are common regional methods for post-thoracotomy pain. In this study, we aimed to compare the efficacy and the side effects of TEA and TPVB methods applied for post-thoracotomy analgesia.

Material and Method: After ethical committee approval, we retrospectively analyzed postoperative analgesia records of patients, who underwent thoracotomy, received TEA or TPVB for postoperative analgesia between 01.01.2019 and 01.01.2021. Visual analog scale (VAS) scores of the patients who received analgesic treatment for 24 hours postoperatively after TEA or TPVB application were evaluated.

Results: Data of 474 patients were found eligible for analysis. Demographic data of patients, VAS scores, patient satisfaction, and additional analgesic requirements were not statistically significant between the groups ( $p>0.05)$. Nausea-vomiting, hypotension, headache, itching, and sweating was statistically significantly higher in the TEA group when compared to patients who underwent TPVB $(\mathrm{p}<0.05)$.

Conclusion: In the present study, in the early postoperative pain management after thoracotomy; it was observed that preemptive TPVB and postoperative IV patient-controlled analgesia (PCA) and TEA provided similar VAS scores and additional analgesic requirements. On the other hand, postoperative complications were observed less frequently in patients who underwent TPVB with postoperative IV PCA compared to TEA. Therefore, TPVB may be a good alternative to prevent acute pain after thoracotomy.
\end{abstract}

Keywords: Pain, postoperative acute pain, thoracic epidural analgesia, thoracic paravertebral block, thoracotomy

\section{INTRODUCTION}

Thoracotomy causes significant trauma to pain-sensitive structures such as muscle layers, fascia, neurovascular bundles, bone, joints, and parietal pleura. For this reason, it is among the most painful surgical incisions and causes severe acute pain in the postoperative period $(1,2)$. Acute pain, on the other hand, increases postoperative morbidity and prolongs hospital stay (3). If thoracotomyrelated pain is not adequately treated, chronic pain may develop after thoracotomy and may prevent patients from regaining their normal activities for a long time $(4,5)$.

Many analgesic methods have been suggested for thoracotomy pain treatment. Some of these are thoracic epidural analgesia (TEA), thoracic paravertebral block (TPVB), plane blocks, intercostal nerve blocks, pleural blocks, as well as systemic and intrathecal analgesics
(6-8). Although the risk of spinal hematoma is not as high as in thoracic epidural catheterization, a normal coagulation profile is also required in block applications (9). TEA is still considered the gold standard technique for post-thoracotomy pain (10-12). The paravertebral space is a wedge-shaped space that extends on either side of the vertebral column and contains formations such as the spinal nerve, dorsal ramus, rami communicantes, and sympathetic chain (6). Administration of local anesthetic to the paravertebral area produces unilateral analgesia, somatic and sympathetic block, and this application is suitable for unilateral surgical procedures of the thoracic region (12). TPVB can be applied unilaterally or bilaterally. TEA affects bilaterally. Therefore, the TPVB technique, unlike TEA, can be used 
to avoid the contralateral sympathetic block. Thus, it minimizes the possibility of hypotension and maintains blood pressure (13). There are studies suggesting that complications such as postoperative nausea-vomiting, hypotension, and urinary retention are less common after TPVB application compared to TEA application $(14,15)$.

In this study, we aimed to compare the efficacy and the side effects of TEA and TPVB methods applied for postthoracotomy analgesia in postoperative acute pain.

\section{MATERIAL AND METHOD}

The study was initiated with the approval of the Keçiören Training and Research Hospital Clinical Researchs Ethics Committee (Date: 11.05.2021, Decision No: 2012-KAEK-15/2307). All procedures were performed adhered to the ethical rules and principles of the Helsinki Declaration.

The data of the patients who had elective thoracic surgery between 01.01.2019 and 01.01.2021 in our clinic were analyzed retrospectively. Patients, who underwent thoracotomy, received TEA or TPVB for postoperative analgesia, were 18-80 years old, and were in the American Society of Anesthesiologists (ASA) I, II, and III physical status. The visual analog scale (VAS) values at the rest of the patients who received analgesic treatment for 24 hours postoperatively after TEA or TPVB application were evaluated. All patients were informed about the application and their informed consent was obtained. We excluded patients who were under the age of 18 , or over the age of 80 , or did not undergo thoracotomy, or were operated under emergency conditions, or had chronic pain before the operation, and constantly used analgesics. Postoperative kinking, catheter dislocation, and occlusion associated with the epidural catheter were defined as "catheter failure". The patients were divided into 2 groups: TPVB and TEA (Figure 1).

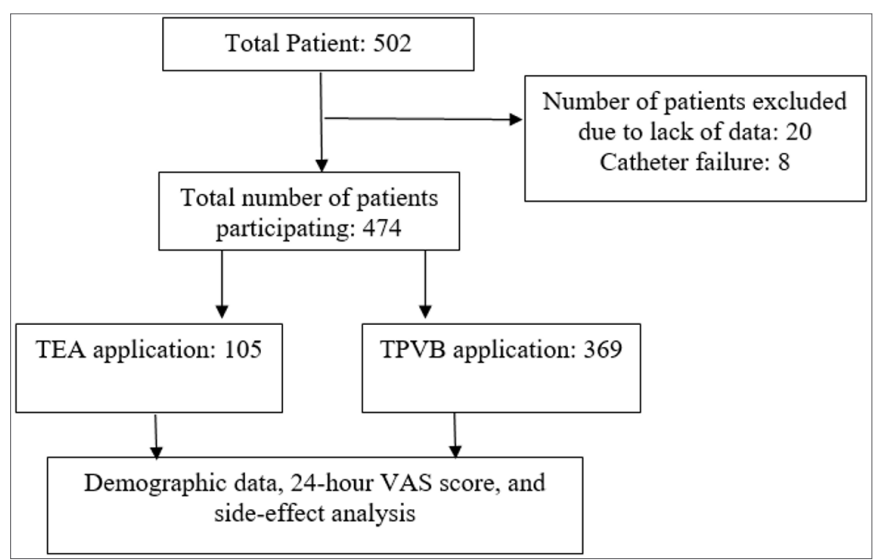

Figure 1. Flow chart of the patients. TEA: Thoracic Epidural Analgesia. TPVB: Thoracic Paravertebral Block.

\section{TEA and TPVB Protocols in Participating Patients}

In the preoperative anesthesia outpatient clinic, TEA and TPVB were explained in detail to the patients who were planned for thoracotomy. If the patients gave consent, TEA was preferred first. Most of the patients preferred TPVB application instead of TEA. Intravenous (IV) patient-controlled analgesia (PCA) was applied to the patients who did not accept either of these methods.

For the patients in the TEA group, in the sitting position, after the skin was cleaned with the strict antisepsis rules and covered, skin anesthesia was performed with $3 \mathrm{ml}$ $2 \%$ prilocaine. The epidural space was entered from the T5-T6 or T6-T7 vertebral spaces with an 18-Gauge Tuohy needle using the median approach and hanging drop method. Four $\mathrm{cm}$ of the catheter was left in the epidural space. To exclude vascular and intrathecal injection, a test dose $(5 \mu \mathrm{g} / \mathrm{ml}(1: 200.000)$ adrenaline and $3 \mathrm{ml} \mathrm{2 \%}$ lidocaine) was administered through the epidural catheter and the patients were placed in the supine position. The bilateral block was evaluated with a pin-prick test. In the intraoperative period; for epidural analgesia, $67.5 \mathrm{ml}$ of $0.5 \%$ bupivacaine, $201.5 \mathrm{ml}$ of saline, and $10 \mathrm{mg} / 1 \mathrm{ml}$ of morphine were mixed with a $270 \mathrm{ml}$ elastomeric infusion pump. A concentration of $0.125 \%$ bupivacaine infusion was started and was given epidurally for 3 days postoperatively, starting with an elastomeric pump at a rate of $4 \mathrm{ml} / \mathrm{h}$. In our study, we considered the first 24-hour follow-up to compare with TPVB.

For general anesthesia induction, $2 \mathrm{mg} / \mathrm{kg}$ propofol, $0.1 \mathrm{mg} / \mathrm{kg}$ vecuronium, and $1 \mu \mathrm{g} / \mathrm{kg}$ fentanyl were administered intravenously (IV). Anesthesia was maintained in both groups with 50\% - 100\% O2 air mixture and $2 \%$ sevoflurane administration with remifentanil infusion $(0.01-0.20 \mathrm{mcg} / \mathrm{kg} / \mathrm{min})$. If the continuation of neuromuscular blockade is necessary, IV $0.03 \mathrm{mg} / \mathrm{kg}$ vecuronium was administered. At the end of the surgery, $50 \mathrm{mg}$ IV dexketoprofen and $100 \mathrm{mg}$ IV tramadol were administered for analgesia, and 10 mg IV metoclopramide as an antiemetic.

For the patients in the TPVB group; under general anesthesia, after the skin was cleaned with strict antisepsis rules and covered in the lateral decubitus position, the ultrasound (US) probe was placed $2-3 \mathrm{~cm}$ lateral to the T5 spinous process just before the surgery. After imaging the transverse process, musculature up to the transverse process, paravertebral area, internal intercostal membrane, and pleura, the needle was advanced to the paravertebral area in the cauda-cranial direction with the in-plane technique. Analgesic treatment of the patients was continued with postoperative IV PCA. Postoperative analgesia was provided by the IV PCA method in the surgical intensive 
care unit. According to our PCA protocol; 400-500 mg of tramadol was added into $100 \mathrm{ml}$ of isotonic sodium chloride. Thus, a concentration of $4-5 \mathrm{mg} / \mathrm{ml}$ tramadol was obtained. It was adjusted to be $10 \mathrm{mg} /$ hour basal infusion, 5-20 mg bolus, 20-30 minutes lock-in time, and 4 hours limit of $100 \mathrm{mg}$. IV PCA treatment was applied for 24 hours.

A paracetamol dose of $1 \mathrm{~g}$ every 8 hours and a dexketoprofen dose of $50 \mathrm{mg}$ twice daily were administered intravenously for multimodal analgesia. $50 \mathrm{mg}$ IV tramadol was given as an "additional analgesic" to patients with a VAS value of 4 and above.

Age, gender, body mass index (BMI), ASA physical status, diagnosis, the type of surgery, duration of anesthesia, VAS values in postoperative 24 hours, analgesic treatment given, additional analgesic need, patient satisfaction, and side effects related to analgesic treatment were recorded. Patient satisfaction was determined as satisfied, moderately satisfied, and dissatisfied.

\section{Statistical Analyses}

Data analyses were performed by using SPSS for Windows, version 22.0 (SPSS Inc., Chicago, IL, United States). Whether the distribution of continuous variables was normal or not was determined by the Kolmogorov Smirnov test. Levene test was used for the evaluation of homogeneity of variances. Unless specified otherwise, continuous data were described as mean \pm standard deviation (SD) and median (minimum-maximum value). Categorical data were described as a number of cases (\%). Statistical analysis differences in not normally distributed variables between two independent groups were compared by the Mann Whitney U test. Categorical variables were compared using Pearson's Chi-Square Test or Fisher's Exact test. It was accepted $\mathrm{p}<0.05$ as a significant level on all statistical analyses.

\section{RESULTS}

The data of 502 patients who underwent elective thoracic surgery and underwent TEA or TPVB between 01.01.2019 and 01.01.2021 were analyzed retrospectively. 28 of these patients were excluded from the study due to a lack of data. Data of 474 patients were found eligible for analysis.

In terms of demographic data when patients were compared, no statistically significant difference was observed in both groups ( $p>0.05)$, (Table 1).

In terms of VAS scores, patient satisfaction, and additional analgesic requirement; there was no statistically significant difference between TEA and TPVB groups ( $>0.05$ ) (Table 2, Figure 2, Figure 3).
Table 1. Demographics Characteristics of Patients

\begin{tabular}{|c|c|c|c|}
\hline & TPVB (n:369) & TEA (n:105) & $\mathbf{p}$ \\
\hline Age & & & 0.835 \\
\hline Mean \pm SD & $53.66 \pm 15.70$ & $55.44 \pm 12.52$ & \\
\hline Median (min-max) & $58(18-80)$ & $56(20-78)$ & \\
\hline Gender & & & 0.058 \\
\hline Female & $116(31.4 \%)$ & $17(21.9 \%)$ & \\
\hline Male & $253(68.6 \%)$ & $88(78.1 \%)$ & \\
\hline BMI & & & 0.07 \\
\hline Mean \pm SD & $26.92 \pm 4.29$ & $26.04 \pm 4.49$ & \\
\hline Median (min-max) & $26.64(18-35)$ & $26.04(18-35)$ & \\
\hline ASA & & & 0.139 \\
\hline ASA II & $123(33.3 \%)$ & $27(25.7 \%)$ & \\
\hline ASA III & $246(66.7 \%)$ & $78(74.3 \%)$ & \\
\hline Diagnosis & & & 0.120 \\
\hline Lung cancer & $283(76.7 \%)$ & $79(75.2 \%)$ & \\
\hline Bronchiectasis & $14(3.8 \%)$ & $11(10.5 \%)$ & \\
\hline Hydatid Cyst & $24(6.5 \%)$ & $6(5.7 \%)$ & \\
\hline Pleural thickening/effusion & $29(7.9 \%)$ & $4(3.8 \%)$ & \\
\hline Interstitial Lung Disease & $2(0.5 \%)$ & - & \\
\hline Other & $17(4.6 \%)$ & $5(4.8 \%)$ & \\
\hline Operation type & & & 0.777 \\
\hline Thoracotomy & $286(77.5 \%)$ & $80(76.2 \%)$ & \\
\hline VATS + Thoracotomy & $83(22.5 \%)$ & $25(23.8 \%)$ & \\
\hline Operation & & & 0.085 \\
\hline Lung Resection & $221(59.9 \%)$ & $7167.6 \%)$ & \\
\hline Pneumonectomy & $37(10 \%)$ & $15(14.3 \%)$ & \\
\hline Exploration-Decortication & $45(12.2 \%)$ & $9(8.6 \%)$ & \\
\hline Cystotomy & $23(6.2 \%)$ & $6(5.7 \%)$ & \\
\hline Other & $43(11.7 \%)$ & $4(3.8 \%)$ & \\
\hline Anesthesia Duration & & & 0.056 \\
\hline Mean \pm SD & $268.2 \pm 72.14$ & $259.5 \pm 29.63$ & \\
\hline Median (min-max) & $270(82-660)$ & $245(190-325)$ & \\
\hline \multicolumn{4}{|c|}{$\begin{array}{l}\text { Continuous variables were expressed as mean } \pm \text { standard deviation }(\mathrm{SD}) \text { and median } \\
\text { (minimum-maximum value). Categorical variables were expressed as frequency } \\
\text { (percentage). Continuous variables were compared with Mann Whitney U-Test, } \\
\text { and categorical variables were compared using Pearson's Chi-Square Test or Fisher's } \\
\text { Exact Test. Statistically significant p }<0.05 \text { are in bold. ASA: American Society of } \\
\text { Anesthesiologists. TPVB: Thoracic Paravertebral Block. TEA: Thoracic Epidural } \\
\text { Analgesia. BMI: Body Mass Index. VATS: Video-Assisted Thoracoscopic Surgery. }\end{array}$} \\
\hline
\end{tabular}

Table 2. Patients' visual analog scale scores, additional analgesic requirements and patient satisfaction

\begin{tabular}{|c|c|c|c|c|}
\hline & & $\begin{array}{l}\text { TPVB } \\
(\mathbf{n}: 369)\end{array}$ & $\begin{array}{c}\text { TEA } \\
(\mathbf{n}: 105)\end{array}$ & $\mathbf{p}$ \\
\hline $\begin{array}{l}\text { VAS } \\
1^{\text {st }} \text { hour }\end{array}$ & $\begin{array}{l}\text { Mean } \pm \text { SD } \\
\text { Median (min-max) }\end{array}$ & $\begin{array}{l}4.12 \pm 1.30 \\
4(0-8)\end{array}$ & $\begin{array}{l}3.90 \pm 1.11 \\
4(2-7)\end{array}$ & 0.061 \\
\hline $\begin{array}{l}\text { VAS } \\
2^{\text {nd }} \text { hour }\end{array}$ & $\begin{array}{l}\text { Mean } \pm \text { SD } \\
\text { Median (min-max) }\end{array}$ & $\begin{array}{c}3.59 \pm 1.21 \\
4(0-7)\end{array}$ & $\begin{array}{c}3.48 \pm 1.02 \\
3(2-6)\end{array}$ & 0.344 \\
\hline $\begin{array}{l}\text { VAS } \\
6^{\text {th }} \text { hour }\end{array}$ & $\begin{array}{l}\text { Mean } \pm \text { SD } \\
\text { Median (min-max) }\end{array}$ & $\begin{array}{c}3.11 \pm 1.14 \\
3(0-7)\end{array}$ & $\begin{array}{c}2.99 \pm 0.95 \\
3(1-6)\end{array}$ & 0.318 \\
\hline $\begin{array}{l}\text { VAS } \\
12^{\text {th }} \text { hour }\end{array}$ & $\begin{array}{l}\text { Mean } \pm \text { SD } \\
\text { Median (min-max) }\end{array}$ & $\begin{array}{l}2.56 \pm 0.89 \\
2(0-6)\end{array}$ & $\begin{array}{c}2.52 \pm 0.82 \\
2(1-5)\end{array}$ & 0.664 \\
\hline $\begin{array}{l}\text { VAS } \\
24^{\text {th }} \text { hour }\end{array}$ & $\begin{array}{l}\text { Mean } \pm \text { SD } \\
\text { Median (min-max) }\end{array}$ & $\begin{array}{c}2.03 \pm 0.93 \\
2(0-5)\end{array}$ & $\begin{array}{c}1.95 \pm 0.79 \\
2(1-4)\end{array}$ & 0.270 \\
\hline \multicolumn{2}{|c|}{$\begin{array}{l}\text { Additional Analgesic } \\
\text { Requirements }\end{array}$} & $\begin{array}{c}84 \\
(22.8 \%)\end{array}$ & $\begin{array}{c}19 \\
(18.1 \%)\end{array}$ & 0.306 \\
\hline \multirow{2}{*}{$\begin{array}{l}\text { Patients } \\
\text { satisfaction }\end{array}$} & Moderately satisfied & $79(21.4 \%)$ & $29(27.6 \%$ & \multirow{2}{*}{0.181} \\
\hline & Satisfied & 290 (78.6\%) & $76(72.4 \%)$ & \\
\hline \multicolumn{5}{|c|}{$\begin{array}{l}\text { Continuous variables were expressed as the mean } \pm \text { standard deviation (SD) and } \\
\text { median (minimum-maximum value, and categorical variables are expressed as either } \\
\text { frequency (percentage). Continuous variables were compared with Mann Whitney } \\
\text { U Test, and categorical variables were compared using Pearson's Chi-Square Test } \\
\text { or Fisher Exact Test. Statistically significant p-values are in bold. TPVB: Thoracic } \\
\text { Paravertebral Block. TEA: Thoracic Epidural Analgesia. VAS: Visual Analog Scale }\end{array}$} \\
\hline
\end{tabular}




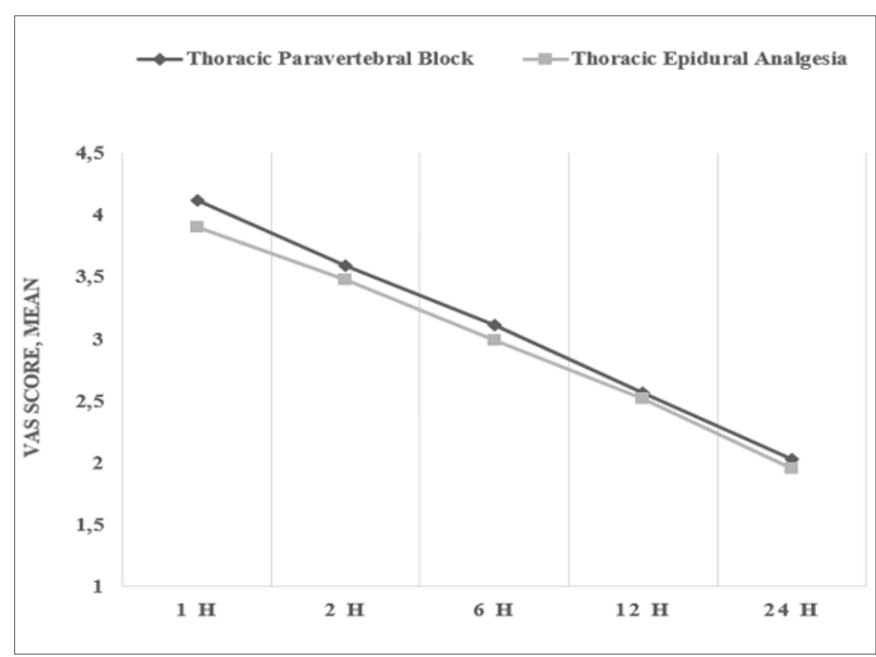

Figure 2. Mean Visual Analog Scale levels of patients over time. VAS: Visual Analog scale.

For patients who underwent TEA; nausea-vomiting, hypotension, headache, itching, and sweating were statistically significantly higher compared to patients who underwent TPVB ( $\mathrm{p}<0.05)$. In terms of bradycardia, there was no statistically significant difference between the groups $(\mathrm{p}>0.05)$, (Table 3$)$.

\begin{tabular}{|lccc|}
\hline \multicolumn{4}{|c|}{ Table 3. Evaluation of the patients in terms of side effects } \\
\hline & TPVB (n:369) & TEA (n:105) & p \\
\hline Nausea/vomiting & $6(1.6 \%)$ & $12(11.4 \%)$ & $<\mathbf{0 . 0 0 1}$ \\
Hypotension & $20(5.4 \%)$ & $13(12.4 \%)$ & $\mathbf{0 . 0 1 3}$ \\
Bradycardia & $1(0.3 \%)$ & $2(1.9 \%)$ & 0.125 \\
Headache & - & $7(6.7 \%)$ & $<\mathbf{0 . 0 0 1}$ \\
Itching & - & $5(4.8 \%)$ & $<\mathbf{0 . 0 0 1}$ \\
Sweating & - & $4(3.8 \%)$ & $<\mathbf{0 . 0 0 1}$ \\
Respiratory depression & - & - & - \\
\hline $\begin{array}{l}\text { Categorical variables were expressed as either frequency (percentage). Variables were } \\
\text { compared using Pearson's Chi-Square Test or Fisher Exact Test. Statistically significant } \\
\text { p-values are in bold. TPVB: Thoracic Paravertebral Block. TEA: Thoracic Epidural } \\
\text { Analgesia. }\end{array}$ \\
\hline
\end{tabular}

\section{DISCUSSION}

In our study, in early postoperative pain management after thoracotomy; we observed that preemptive TPVB and postoperative IV PCA application, and TEA administered with postoperative fixed infusion provided similar VAS scores and additional analgesic requirements. On the other hand, postoperative complications were observed less frequently in patients who underwent TPVB and IV PCA compared to TEA.

Thoracotomy is among the most painful surgical procedure. If not treated effectively, it increases postoperative pulmonary complications such as painrelated atelectasis, pulmonary embolism, and pneumonia in the early postoperative period (1). As a result, it increases postoperative morbidity and prolongs hospital stay (2). Another significant problem after thoracotomy is the development of chronic pain, and this may prevent

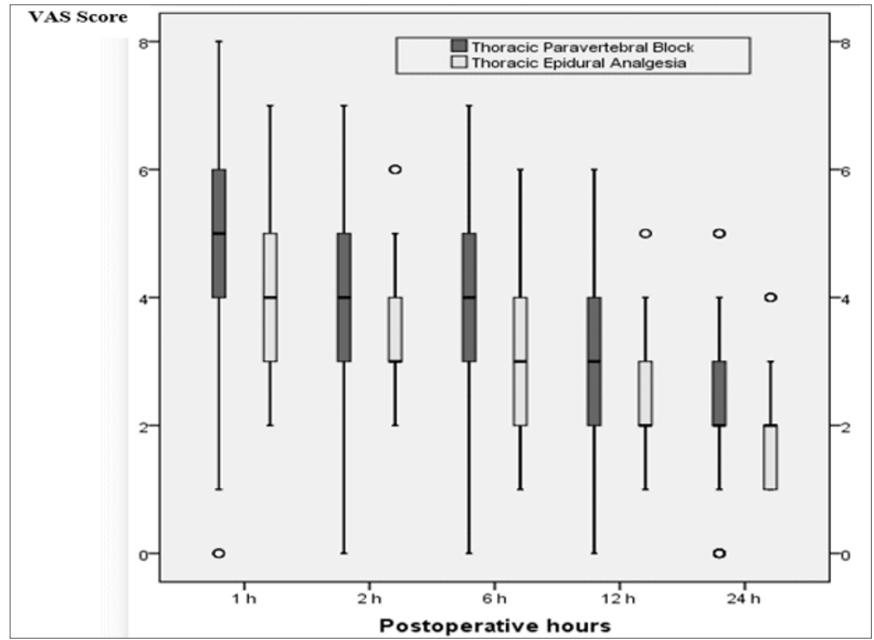

Figure 3. Median, minimum and maximum Visual Analog Scale levels of patients over time. VAS: Visual Analog Scale.

patients from regaining their normal activities for a long time $(3,4)$. Therefore, effective perioperative analgesia is one of the most vital elements in thoracotomy $(6,7)$. Effective postoperative analgesia is the method in which multimodal techniques are applied. Multimodal analgesia provides both comprehensive analgesias and limits the side effects via lower dose usage of opioid analgesics. One of the most important components of multimodal analgesia is regional techniques (6-8). TEA and TPVB are regional methods that have been used for many years in pain after thoracotomy $(10,11)$.

TEA is a regional analgesia method that is still used as the gold standard for post-thoracotomy pain. There are studies showing that TPVB, which has been widely applied in recent years, provides a similar or even more effective analgesic effect than TEA (12,16-19). The analgesic effect due to TPVB occurs with unilateral somatic and sympathetic block effects. By providing a block in the upper and lower dermatomal area in the application area, pain levels that may occur along the thoracotomy line can be achieved as in TEA $(12,19)$. In our study, it was found that similar pain scores were obtained with TEA and the rates of additional analgesics used were similar. These results are consistent with the literature and we think that TPVB is an effective analgesic method in acute pain after thoracotomy.

Undesirable complications such as hypotension, bradycardia, nausea-vomiting, and urinary retention that may occur due to sympathetic block after TEA limit its use $(13,20)$. In addition; the increase in the use of anticoagulants due to the increase in the elderly patient population and cancer surgery applications in recent years has led to alternative methods due to catastrophic complications such as epidural hematoma that may develop after TEA $(6,21,22)$. The most frequently used of these applications is the TPVB application. In TPVB, the somatic effect due to the block is prominent and a limited 
level of sympathetic block develops. In addition, it is safe in terms of avoiding epidural hematoma and similar complications due to its distance from the epidural area $(14,15,17,18)$. In our study, complication rates were also quite limited in the TPVB group compared to TEA, and this shows that TPVB can be used safely in postthoracotomy pain.

Acceleration of postoperative recovery (ERAS) protocols, which have become an important issue in thoracic surgery in recent years, especially support opioidfree and low complication rate analgesia protocols $(23,24)$. The purpose of ERAS protocols is to reduce the complication rate by making the least intervention with the least complication and to ensure that the patients are discharged quickly and without problems $(25,26)$. It is claimed that paravertebral blockade provides analgesia equivalent to epidural analgesia (27). In our study, only local anesthetic administration and postoperative analgesia with tramadol, a weak opioid, in TPVB application may be a suitable alternative in the ERAS protocol, since it reduces the complications that may develop with potent opioids.

We have some limitations in our study. First of all, our study is single-center and retrospective. In addition, the effects of these two methods on chronic pain could not be evaluated because the long-term records of the patients could not be reached.

\section{CONCLUSION}

In our study, in early postoperative pain management after thoracotomy; it was observed that preemptive TPVB with postoperative IV PCA application and TEA provided similar VAS scores and additional analgesic requirements. On the other hand, postoperative complications were observed less frequently in patients who underwent TPVB and postoperative IV PCA compared to TEA. As stated in the ERAS protocols, TPVB can provide equivalent analgesia to TEA. Therefore, TPVB may be a good alternative to prevent acute pain in the post-thoracotomy period.

\section{ETHICAL DECLARATION}

Ethics Committee Approval: The study was initiated with the approval of the Keçiören Training and Research Hospital Clinical Researchs Ethics Committee (Date: 11.05.2021, Decision No: 2012-KEAK-15/2307).

Informed Consent: Because the study was designed retrospectively, no written informed consent form was obtained from patients.

Referee Evaluation Process: Externally peer-reviewed.
Conflict of Interest Statement: The authors have no conflicts of interest to declare.

Financial Disclosure: The authors declared that this study has received no financial support.

Author Contributions: All of the authors declare that they have all participated in the design, execution, and analysis of the paper and that they have approved the final version.

\section{REFERENCES}

1. Tamura T, Mori S, Mori A, et al. A randomized controlled trial comparing paravertebral block via the surgical field with thoracic epidural block using ropivacaine for post-thoracotomy pain relief. J Anesth 2017; 31: 263-70.

2. Sentürk M, Ozcan PE, Talu GK, et al. The effects of three different analgesia techniques on long-term postthoracotomy pain. Anesth Analg 2002; 94: 11-5.

3. Khalil AE, Abdallah NM, Bashandy GM, Kaddah TA. Ultrasound-guided serratus anterior plane block versus thoracic epidural analgesia for thoracotomy pain. J Cardiothorac Vasc Anesth 2017; 31: 152-8.

4. Ochroch EA, Gottschalk A. Impact of acute pain and its management for thoracic surgical patients. Thorac Surg Clin 2005; 15: 105-21.

5. Rogers ML, Henderson L, Mahajan RP, Duffy JP. Preliminary findings in the neurophysiological assessment of intercostal nerve injury during thoracotomy. Eur J Cardiothorac Surg 2002; 21: 298-301.

6. Davies RG, Myles PS, Graham JM. A comparison of the analgesic efficacy and side-effects of paravertebral vs epidural blockade for thoracotomy--a systematic review and meta-analysis of randomized trials. Br J Anaesth 2006; 96: 418-26.

7. Joshi GP, Bonnet F, Shah R, et al. A systematic review of randomized trials evaluating regional techniques for postthoracotomy analgesia. Anesth Analg 2008; 107: 1026-40.

8. Schnabel A, Reichl SU, Kranke P, Pogatzki-Zahn EM, Zahn PK. Efficacy and safety of paravertebral blocks in breast surgery: a meta-analysis of randomized controlled trials. Br J Anaesth 2010; 105: 842-52.

9. Madabushi R, Tewari S, Gautam SK, Agarwal A, Agarwal A. Serratus anterior plane block: a new analgesic technique for postthoracotomy pain. Pain Physician 2015; 18: E421-4.

10. Barbera C, Milito P, Punturieri M, Asti E, Bonavina L. Serratus anterior plane block for hybrid transthoracic esophagectomy: a pilot study. J Pain Res 2017; 4: 73-7.

11. Özden Omaygenç D, Çıtak N, İşgörücü Ö, et al. Comparison of thoracic epidural and intravenous analgesia from the perspective of recovery of respiratory function in the early post-thoracotomy period in lung cancer surgery. Turk Thorac J 2021; 22: 31-6.

12. Karmakar MK. Thoracic paravertebral block. Anesthesiology 2001; 95: 771-80.

13. D’Ercole F, Arora H, Kumar PA. Paravertebral block for thoracic surgery. J Cardiothorac Vasc Anesth 2018; 32: 915-27.

14. Fang B, Wang Z, Huang X. Ultrasound-guided preoperative single-dose erector spinae plane block provides comparable analgesia to thoracic paravertebral block following thoracotomy: a single center randomized controlled double-blind study. Ann Transl Med 2019; 7: 174.

15. Wojtyś ME, Wąsikowski J, Wójcik N, et al. Assessment of postoperative pain management and comparison of effectiveness of pain relief treatment involving paravertebral block and thoracic epidural analgesia in patients undergoing posterolateral thoracotomy. J Cardiothorac Surg 2019; 14: 78. 
16. Yeung JH, Gates S, Naidu BV, Wilson MJ, Gao Smith F. Paravertebral block versus thoracic epidural for patients undergoing thoracotomy. Cochrane Database Syst Rev 2016; 2: CD009121.

17. Detterbeck FC. Efficacy of methods of intercostal nerve blockade for pain relief after thoracotomy. Ann Thorac Surg 2005; 80: $1550-9$.

18. Kotzé A, Scally A, Howell S. Efficacy and safety of different techniques of paravertebral block for analgesia after thoracotomy: a systematic review and metaregression. Br J Anaesth 2009; 103: 626-36.

19. Richardson J, Sabanathan S, Jones J, Shah RD, Cheema S, Mearns AJ. A prospective, randomized comparison of preoperative and continuous balanced epidural or paravertebral bupivacaine on post-thoracotomy pain, pulmonary function and stress responses. Br J Anaesth 1999; 83: 387-92.

20. Kupersztych-Hagege E, Dubuisson E, Szekely B, et al. Epidural hematoma and abscess related to thoracic epidural analgesia: a single-center study of 2,907 patients who underwent lung surgery. J Cardiothorac Vasc Anesth 2017; 31: 446-52.

21. El Shora HA, El Beleehy AA, Abdelwahab AA, et al. Bilateral paravertebral block versus thoracic epidural analgesia for pain control post-cardiac surgery: a randomized controlled trial Thorac Cardiovasc Surg 2020; 68: 410-6.

22. Conlon NP, Shaw AD, Grichnik KP. Postthoracotomy paravertebral analgesia: will it replace epidural analgesia? Anesthesiol Clin 2008; 26: 369-80.

23. Simpson JC, Bao X, Agarwala A. Pain management in enhanced recovery after surgery (ERAS) protocols. Clin Colon Rectal Surg 2019; 32: 121-8.

24. Gupta R, Van de Ven T, Pyati S. Post-thoracotomy pain: current strategies for prevention and treatment. Drugs 2020; 80: 1677-84.

25. Batchelor TJP, Ljungqvist O. A surgical perspective of ERAS guidelines in thoracic surgery. Curr Opin Anaesthesiol 2019; 32: 17-22.

26. Nicholson A, Lowe MC, Parker J, Lewis SR, Alderson P, Smith AF. Systematic review and meta-analysis of enhanced recovery programmes in surgical patients. Br J Surg 2014; 101: 172-88.

27. Batchelor TJP, Rasburn NJ, Abdelnour-Berchtold E, et al. Guidelines for enhanced recovery after lung surgery: recommendations of the Enhanced Recovery After Surgery (ERAS ${ }^{\circ}$ ) Society and the European Society of Thoracic Surgeons (ESTS). Eur J Cardiothorac Surg 2019; 55: 91-115. 